\title{
An Adaptive Finite Element PML Method for the Acoustic Scattering Problems in Layered Media
}

\author{
Xue Jiang $^{1, *}$, Yu Qi ${ }^{1}$ and Jianhua Yuan ${ }^{1}$ \\ 1 School of Science, Beijing University of Posts and Telecommunications, Beijing \\ 100876, China.
}

Received 15 February 2018; Accepted (in revised version) 11 April 2018

\begin{abstract}
The paper concerns the numerical solution for the acoustic scattering problems in a two-layer medium. The perfectly matched layer (PML) technique is adopted to truncate the unbounded physical domain into a bounded computational domain. An a posteriori error estimate based adaptive finite element method is developed to solve the scattering problem. Numerical experiments are included to demonstrate the efficiency of the proposed method.
\end{abstract}

AMS subject classifications: 65N30, 78M10, 35Q99

Key words: Acoustic scattering problems, layered media, perfectly matched layer, adaptive finite element method.

\section{Introduction}

Numerical solutions of scattering problems have drawn considerable attention in both the engineering and mathematical communities. The first key point of numerical solutions is the treatment of radiation conditions at infinity. It involves the truncation of an unbounded domain to a bounded domain and imposes highly accurate boundary conditions at the artificial boundary (cf. e.g. [24-26, 40]). Scattering problems involving infinite boundaries, such as the scattering in layered media and half-spaces (cf. e.g. $[9,17,20,22,41])$, are studied recently. With the appearance of infinite boundaries, the scattering waves usually comprise reflective waves and evanescent waves. Hence the numerical treatment of radiation conditions becomes very challenging and appeals for new theories and methods.

*Corresponding author. Email addresses: jxue@lsec.cc.ac.cn (X. Jiang), cynthia18@vip.qq.com (Y. Qi), jianhuayuan@bupt.edu.cn (J. Yuan) 


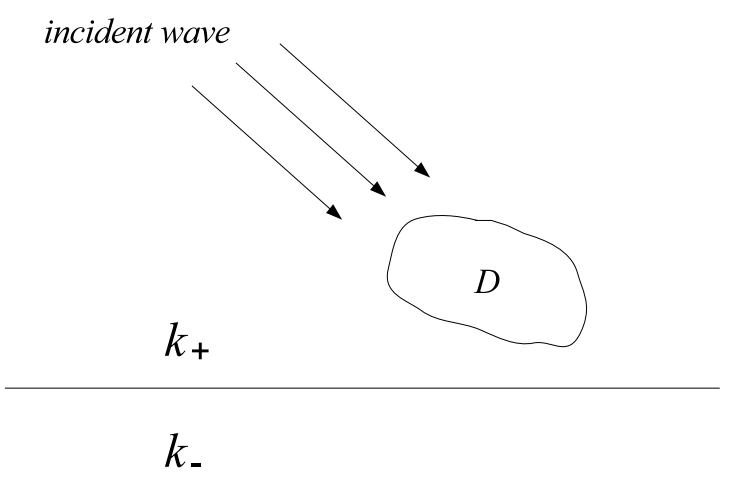

Figure 1: A schematic of the geometry for the scattering problem in a two-layer media.

In this paper, we study the two-dimensional acoustic scattering problems in a twolayer medium:

$$
\begin{aligned}
\Delta u+k^{2} u & =0 & & \text { in } \mathbb{R}^{2} \backslash \bar{D}, \\
u & =g & & \text { on } \Gamma_{D}, \\
{[u]=\left[\frac{\partial u}{\partial x_{2}}\right] } & =0 & & \text { on } \Sigma, \\
\sqrt{r}\left(\frac{\partial u}{\partial r}-\mathrm{i} k u\right) & \rightarrow 0 & & \text { as } r=|x| \rightarrow \infty,
\end{aligned}
$$

where $D \subset \mathbb{R}^{2}$ is a bounded domain with Lipschitz boundary $\Gamma_{D}$ and $g \in H^{1 / 2}\left(\Gamma_{D}\right), u$ is the scattering field, $\Sigma=\left\{\left(x_{1}, x_{2}\right) \in \mathbb{R}^{2}: x_{2}=0\right\}$ is the interface, and $[u]_{\Sigma}:=u_{+}-u_{-}$is the jump of $u$ across $\Sigma$ from above to below. We assume the wave number $k$ is positive and piecewise constant, defined by

$$
k(x)= \begin{cases}k_{+}, & \text {if } x \in \mathbb{R}_{+}^{2}, \\ k_{-}, & \text {if } x \in \mathbb{R}_{-}^{2},\end{cases}
$$

where $\mathbb{R}_{ \pm}^{2}=\left\{\left(x_{1}, x_{2}\right) \in \mathbb{R}^{2}: \pm x_{2}>0\right\}$. Without loss of generality we assume in this paper that $k_{-}>k_{+}>0$. We consider an acoustic incident wave in a two-layer medium. Due to the existence of $\Sigma$, the scattering waves consist of both propagating modes and evanescent modes. The problem geometry is shown in Fig. 1.

The PML method was first proposed by Bérénger [3] for solving the time dependent Maxwell equations. Following this, various constructions of PML absorbing layers have been proposed and studied in the literature. A detailed review of these methods can be found in Turkel and Yefet [39], Teixeira and Chew [38]. The basic idea of the PML method is to surround the computational domain by a layer of finite thickness with specially designed model medium that absorbs all the waves that propagate from inside the computational domain. Bao and $\mathrm{Wu}$ first proved the exponential convergence of the PML 
method for time-harmonic Maxwell's equations in 2005 [2]. The convergence of the PML method for homogeneous background materials has been well-studied, cf., [27,33,34] for circular PML methods for acoustic scattering problems, $[2,10]$ for circular PML methods for electromagnetic scattering problems, and $[4,11]$ for UPML methods. It is proven that the PML solution converges exponentially to the solution of the original scattering problem as the thickness of the PML layer tends to infinity. In [4-7], Bramble and Pasciak studied the stability and exponential convergence of the PML method in both circular and Cartesian coordinates for acoustic and electromagnetic scattering problems. In [35], Liang and Xiang studied the convergence of an anisotropic PML method for Helmholtz scattering problems. We also refer the reader to the papers $[8,15,29,31]$ on the PML methods for elastic scattering problems. The analysis for two-layer media is very challenging for scattering by both propagating waves and evanescent waves. In [14,16], Chen and Zheng proved the exponential convergence of the PML method for time-harmonic acoustic and electromagnetic scattering problems in two-layer media. The main objective of this paper is to study the adaptive finite element PML method for the scattering problems in layered media.

In the practical application of PML methods, Chen and coauthors developed the adaptive PML method for solving acoustic and electromagnetic scattering problems [1013]. The adaptive PML method provides a complete numerical strategy to solve the scattering problems using finite elements. It produces automatically a coarse mesh size away from the fixed domain and thus makes the total computational costs insensitive to the thickness of the PML absorbing layer. The main idea of the method is to use the a posteriori error estimate to determine the PML parameters and to use the adaptive finite element method to solve the PML equations. In [28], Jiang and Zheng proposed an efficient adaptive finite element algorithm to solve the multiple scattering problem. The computation effort is comparable to that of single scattering problems. In [30], Jiang and Li studied the adaptive PML method for the time-harmonic acoustic-elastic interaction problem in three dimensions. In the literature, there is rare work on the adaptive finite element PML method for the scattering problems in layered media and on rigorous a posteriori error estimate.

This paper aims to investigate the adaptive finite element PML method for solving the acoustic scattering problems in a two-layer medium. The PML technique is adopted to truncate the unbounded physical domain into a bounded computational domain. To efficiently resolve the solution with possible singularities, the a posteriori error estimate based on adaptive finite element method is developed to solve the truncated PML problem. The error estimate consists of the PML error and the finite element discretization error, and provides a theoretical basis for the mesh refinement. Numerical experiments are reported to show the competitive behavior of the proposed method.

The paper is organized as follows. In Section 2, we introduce the model equations for the acoustic scattering problems in a two-layer medium. In Section 3, we present the PML formulation on the truncated domain, and the exponential convergence of the PML problem. In Section 4, we introduce the conforming finite element approximation to the 
PML problem. Reliable a posteriori error estimate is derived to control both the thickness of the PML and the mesh refinements. In Section 5, we propose an APML algorithm based on reliable a posteriori error estimates, and show some numerical experiments.

\section{Problem formulation}

In (1.1), the model equations for acoustic scattering in two-layer media is proposed. In this section, an exact transparent boundary condition is introduced to reformulate the scattering problem into a boundary value problem in a bounded domain. Then we propose a weak formulation of the scattering problem (1.1) in the bounded domain.

Let $B=\left\{\boldsymbol{x}=\left(x_{1}, x_{2}\right) \in \mathbb{R}^{2}:\left|x_{j}\right|<L_{j}, j=1,2\right\}$ be a rectangular box which encloses all scatterers and inhomogeneities of the medium. Let $\Omega$ be a bounded Lipschitz domain, and let $\Gamma$ be the boundary of $\Omega$. Denote by $L^{2}(\Omega)$ the usual Hilbert space of square integrable functions. The space is equipped with the following inner product and norm

$$
(u, v)=\int_{\Omega} u(x) \bar{v}(x) \mathrm{d} x \text { and }\|u\|_{L^{2}(\Omega)}=(u, u)^{1 / 2},
$$

where $\bar{v}$ denotes the complex conjugate of $v$. Let $H^{1}(\Omega)$ be the standard Sobolev space equipped with the norm

$$
\|u\|_{H^{1}(\Omega)}=\left(\|u\|_{L^{2}(\Omega)}^{2}+\|\nabla u\|_{L^{2}(\Omega)}^{2}\right)^{1 / 2} .
$$

For any $\Lambda \subset \Gamma$, the subspace with zero trace on $\Lambda$ is denoted by $H_{\Lambda}^{1}(\Omega):=\left\{v \in H^{1}(\Omega): v=\right.$ 0on $\Lambda\}$. In particular, we use the conventional notation $H_{0}^{1}(\Omega):=\left\{v \in H^{1}(\Omega): v=0\right.$ on $\left.\Gamma\right\}$.

The trace space $H^{s}(\Gamma)$ is defined by

$$
H^{s}(\Gamma)=\left\{u \in L^{2}(\Gamma):\|u\|_{H^{s}(\Gamma)}<\infty\right\} .
$$

It is clear to note that the dual space of $H^{s}(\Gamma)$ is $H^{-s}(\Gamma)$ with respect to the scalar product in $L^{2}(\Gamma)$ defined by

$$
\langle u, v\rangle_{\Gamma}=\int_{\Gamma} u \bar{v} \mathrm{~d} s
$$

Now we introduce the Dirichlet-to-Neumann (DtN) operator $\mathscr{T}: H^{1 / 2}\left(\Gamma_{B}\right) \rightarrow H^{-1 / 2}\left(\Gamma_{B}\right)$, where $\Gamma_{B}$ is the boundary of $B$. Given $v \in H^{1 / 2}\left(\Gamma_{B}\right)$, we define

$$
\mathscr{T} v=\partial_{n} u \quad \text { on } \Gamma_{B} \text {, }
$$

where $u$ is the solution of the exterior Dirichlet problem of the Helmholtz equation:

$$
\begin{aligned}
\Delta u+k^{2} u & =0 & & \text { in } \mathbb{R}^{3} \backslash \bar{B}, \\
u & =v & & \text { on } \Gamma_{B}, \\
{[u]=\left[\frac{\partial u}{\partial x_{2}}\right] } & =0 & & \text { on } \Sigma, \\
\sqrt{r}\left(\frac{\partial u}{\partial r}-\mathrm{i} k u\right) & \rightarrow 0 & & \text { as } r=|x| \rightarrow \infty .
\end{aligned}
$$


The well-posedness of the exterior problem (2.2) is proved by Theorem $3.1 \mathrm{in} \mathrm{[14].} \mathrm{There-}$ fore the DtN operator $\mathscr{T}: H^{1 / 2}\left(\Gamma_{B}\right) \rightarrow H^{-1 / 2}\left(\Gamma_{B}\right)$ is well-defined and is a bounded linear operator.

Using the DtN operator $\mathscr{T}$, we reformulate the boundary value problem (1.1) from the open domain into the bounded domain: Given $g \in H^{1 / 2}\left(\Gamma_{D}\right)$, find $u$ such that

$$
\begin{aligned}
\Delta u+k^{2} u=0 & \text { in } \Omega:=B \backslash \bar{D}, \\
u=g & \text { on } \Gamma_{D}, \\
{[u]=\left[\frac{\partial u}{\partial x_{2}}\right]=0 } & \text { on } \Sigma \cap \Omega, \\
\partial_{n} u=\mathscr{T} u & \text { on } \Gamma_{B} .
\end{aligned}
$$

Let $a: H^{1}(\Omega) \times H^{1}(\Omega) \rightarrow \mathbb{C}$ be the sesquilinear form:

$$
a(u, v)=\int_{\Omega}\left(\nabla u \cdot \nabla \bar{v}-k^{2} u \bar{v}\right) \mathrm{d} x-\int_{\Gamma_{B}}(\mathscr{T} u) \bar{v} \mathrm{~d} s .
$$

Then the scattering problem (1.1) is equivalent to the following weak formulation: Find $u \in H^{1}(\Omega)$ such that $u=g$ on $\Gamma_{D}$, and

$$
a(u, v)=0, \quad \forall v \in H_{\Gamma_{D}}^{1}(\Omega) .
$$

For any $g \in H^{1 / 2}\left(\Gamma_{D}\right)$, the scattering problem (1.1) has a unique solution $u \in H_{\text {loc }}^{1}\left(\mathbb{R}^{2} \backslash \bar{D}\right)$, which can be proved by using integral method in Colton and Kress [19, Chap. 3], and similar argument as that in [32]. By the well-posedness of the problem (1.1), the variational problem (2.5) has a unique weak solution $u \in H^{1}(\Omega)$. Then the general theory in Babuška and Aziz [1, Chap. 5] implies that there exists a constant $\gamma$ such that the following inf-sup condition is satisfied

$$
\sup _{0 \neq v \in H^{1}(\Omega)} \frac{|a(u, v)|}{\|v\|_{H^{1}(\Omega)}} \geq \gamma\|u\|_{H^{1}(\Omega)}, \quad \forall v \in H^{1}(B) .
$$

\section{The PML problem}

In this section, we introduce the PML formulation for the scattering problem, and the transparent boundary condition of the PML problem.

\subsection{PML formulation}

First, we introduce an uniaxial absorbing PML layer. Let $B_{1}=\left\{\boldsymbol{x}=\left(x_{1}, x_{2}\right) \in \mathbb{R}^{2}:\left|x_{j}\right|<\right.$ $\left.L_{j}+d_{j}, j=1,2\right\}$ be a larger rectangular box which contains $B$. As is shown in Fig. 2 , the domain $\Omega$ is surrounded by a PML layer of thickness $d_{j}, j=1,2$, which is denoted as 


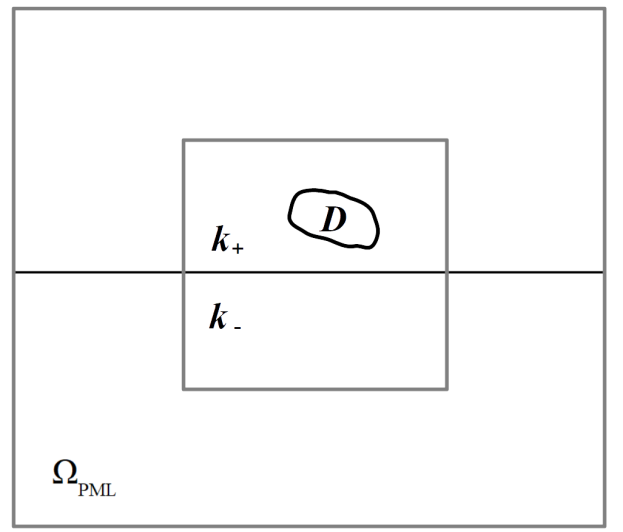

Figure 2: A schematic of the geometry for the PML problem.

$\Omega_{\mathrm{PML}}:=B_{1} \backslash \bar{B}$. Let $\alpha_{j}(t)=1+\mathrm{i} \sigma_{j}(t), j=1,2$, be the PML function which is continuous and satisfies

$$
\sigma_{j}(t)=0 \quad \text { for }|t|<L_{j} \quad \text { and } \quad \sigma_{j}(t)>0 \quad \text { otherwise. }
$$

Following [18], we introduce the PML by the complex coordinate stretching:

$$
\tilde{x}_{j}=\int_{0}^{x_{j}} \alpha_{j}(\tau) \mathrm{d} \tau, \quad j=1,2 .
$$

Notice that $\tilde{x}_{j}$ depends only on $x_{j}$ and for this reason the method is called the uniaxial PML method.

For convenience of theoretical analysis, we make the following assumption on the fictitious medium property which is rather mild in practical applications of the UPML method:

(H1) $\sigma_{j}(t)=\sigma>0, \quad \forall|t| \geq L_{j}, j=1,2$, where $\sigma$ is a positive constant.

(H2) $\int_{0}^{L_{1}+d_{1}} \sigma_{1}(t) \mathrm{d} t=\int_{0}^{L_{2}+d_{2}} \sigma_{2}(t) \mathrm{d} t=: \bar{\sigma}, \quad \bar{\sigma} \geq 1$ is a constant.

Let $\tilde{u}(x)=u(\tilde{x})$ be the PML extension of the solution $u$ of the scattering problem (2.5). It is obvious that $\tilde{u}$ satisfies

$$
\frac{\partial^{2} \tilde{u}}{\partial \tilde{x}_{1}^{2}}+\frac{\partial^{2} \tilde{u}}{\partial \tilde{x}_{2}^{2}}+k^{2} \tilde{u}=0 \quad \text { in } \mathbb{R}^{2} \backslash \bar{D},
$$

which yields the desired UPML equation by the chain rule

$$
\nabla \cdot(A \nabla \tilde{u})+b k^{2} \tilde{u}=0 \quad \text { in } \mathbb{R}^{2} \backslash \bar{D},
$$


where $A=\operatorname{diag}\left(\alpha_{2}\left(x_{2}\right) / \alpha_{1}\left(x_{1}\right), \alpha_{1}\left(x_{1}\right) / \alpha_{2}\left(x_{2}\right)\right)$ is a diagonal matrix, and $b=\alpha_{1}\left(x_{1}\right) \alpha_{2}\left(x_{2}\right)$.

We define $\rho(\tilde{x}, y):=\left(z_{1}^{2}+z_{2}^{2}\right)^{1 / 2}$, where

$$
z_{1}=\left[\left(\tilde{x}_{1}-y_{1}\right)^{2}\right]^{1 / 2}, \quad z_{2}=\left[\left(\tilde{x}_{2}\right)^{2}\right]^{1 / 2}+\left|y_{2}\right|, \quad \forall x, y \in \mathbb{R}^{2}
$$

By the following elementary Lemma 3.1 and (H2), we find that, for any $x \in \Gamma_{B_{1}}, y \in \Gamma_{B}$,

$$
\operatorname{Im} \rho(\tilde{x}, \boldsymbol{y}) \geq \gamma_{0} \bar{\sigma}, \quad \gamma_{0}=\frac{\min \left(d_{1}, d_{2}+L_{2}\right)}{\sqrt{\left(2 L_{1}+d_{1}\right)^{2}+\left(2 L_{2}+d_{2}\right)^{2}}}
$$

Lemma 3.1. ([13, Lemma 3.2]) For any $\tilde{a}=a_{1}+\mathbf{i} a_{2}$ and $\tilde{b}=b_{1}+\mathbf{i} b_{2}$ with $a_{1}, a_{2}, b_{1}, b_{2} \in \mathbb{R}$ such that $a_{1} a_{2}+b_{1} b_{2}>0$ and $a_{1}^{2}+b_{1}^{2}>0$, we have

$$
\operatorname{Im}\left(\tilde{a}^{2}+\tilde{b}^{2}\right)^{1 / 2} \geq \frac{a_{1} a_{2}+b_{1} b_{2}}{\sqrt{a_{1}^{2}+b_{1}^{2}}} .
$$

The outgoing wave $\tilde{u}(x)$ decays exponentially in the PML layer. Therefore, the homogeneous Dirichlet boundary condition is imposed on $\Gamma_{B_{1}}$ to truncate the PML problem. We arrive at the following truncated PML problem: Find $\hat{u}$ such that

$$
\begin{array}{rlrl}
\nabla \cdot(A \nabla \hat{u})+b k^{2} \hat{u} & =0 & & \text { in } \Omega_{1}:=B_{1} \backslash \bar{D}, \\
\hat{u} & =g & & \text { on } \Gamma_{D}, \\
{[\hat{u}]=\left[\frac{\partial \hat{u}}{\partial x_{2}}\right]=0} & & \text { on } \Sigma \cap \Omega_{1}, \\
\hat{u} & =0 & & \text { on } \Gamma_{B_{1}} .
\end{array}
$$

The weak formulation of the truncated PML problem (3.3) reads as follows: Find $\hat{u} \in H_{\Gamma_{B_{1}}}^{1}\left(\Omega_{1}\right)$ such that $\hat{u}=g$ on $\Gamma_{D}$, and

$$
b(\hat{u}, v)=0, \quad \forall v \in H_{0}^{1}\left(\Omega_{1}\right),
$$

where the sesquilinear form $b: H^{1}\left(\Omega_{1}\right) \times H^{1}\left(\Omega_{1}\right)$ is defined by

$$
b(u, v)=\int_{\Omega}\left(A \nabla u \cdot \nabla \bar{v}-k^{2} b u \bar{v}\right) \mathrm{d} x .
$$

In Section 3.3, we will present the exponential convergence of the solution of PML problem (3.3) to the solution of the original scattering problem. 


\subsection{PML problem in the layer}

Given $\xi \in H^{1 / 2}\left(\Gamma_{B}\right)$, consider the following boundary value problem in the PML layer:

$$
\begin{aligned}
\nabla \cdot(A \nabla \varphi)+b k^{2} \varphi & =0 & & \text { in } \Omega_{\mathrm{PML}}, \\
\varphi & =\xi & & \text { on } \Gamma_{B}, \\
{[\varphi]=\left[\frac{\partial \varphi}{\partial x_{2}}\right] } & =0 & & \text { on } \Sigma \cap \Omega_{\mathrm{PML}}, \\
\varphi & =0 & & \text { on } \Gamma_{B_{1}} .
\end{aligned}
$$

Introduce the sesquilinear form $c: H^{1}\left(\Omega_{\mathrm{PML}}\right) \times H^{1}\left(\Omega_{\mathrm{PML}}\right) \rightarrow \mathbb{C}$ as follows:

$$
c(\varphi, \psi)=\int_{\Omega_{\mathrm{PML}}}\left(A \nabla \varphi \cdot \nabla \bar{\psi}-b k^{2} \varphi \bar{\psi}\right) \mathrm{d} x .
$$

The weak formulation for (3.5) is: Given $\xi \in H^{1 / 2}\left(\Gamma_{B}\right)$, find $\varphi \in H^{1}\left(\Omega_{\mathrm{PML}}\right)$ such that $\varphi=0$ on $\Gamma_{B_{1}}, \varphi=\xi$ on $\Gamma_{B}$, and

$$
c(\varphi, \psi)=0, \quad \forall \psi \in H_{0}^{1}\left(\Omega_{\mathrm{PML}}\right) .
$$

In order to obtain a constant independent of PML parameter $\sigma$ in the inf-sup condition, we define

$$
\|\| \varphi \|_{\Omega_{\mathrm{PML}}}=\left(\frac{1}{1+\sigma^{2}}\|\nabla \varphi\|_{L^{2}\left(\Omega_{\mathrm{PML}}\right)}^{2}+\left(1+\sigma^{2}\right)\|k \varphi\|_{L^{2}\left(\Omega_{\mathrm{PML}}\right)}^{2}\right)^{1 / 2} .
$$

By [14, Lemma 5.1], we know that (3.6) has a unique solution and it holds that

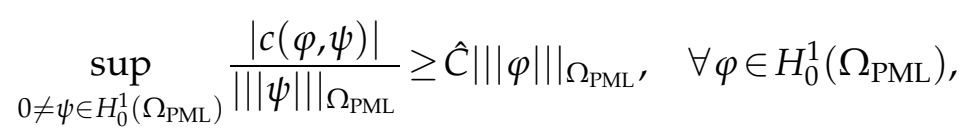

where

$$
\hat{C}=\frac{\min \left(1, \sigma^{3}\right)}{2\left(1+\sigma^{2}\right)^{2} \max \left(1, k_{-}^{2} d^{2}\right)}, \quad d=\max \left(d_{1}, d_{2}\right) .
$$

For any $\varphi \in H^{1}(\Omega)$, let $\tilde{\varphi}$ be its extension in $\Omega_{\mathrm{PML}}$ such that

$$
\begin{aligned}
\nabla \cdot(\bar{A} \nabla \tilde{\varphi})+\bar{b} k^{2} \tilde{\varphi} & =0 & & \text { in } \Omega_{\mathrm{PML}} \\
\tilde{\varphi} & =\varphi & & \text { on } \Gamma_{B}, \\
{[\tilde{\varphi}]=\left[\frac{\partial \tilde{\varphi}}{\partial x_{2}}\right] } & =0 & & \text { on } \Sigma \cap \Omega_{\mathrm{PML}}, \\
\varphi & =0 & & \text { on } \Gamma_{B_{1}} .
\end{aligned}
$$


Lemma 3.2 (Estimates for the extension). For any $\varphi \in H^{1}(\Omega)$, which is extended to be a function $\tilde{\varphi} \in H^{1}\left(\Omega_{1}\right)$ according to (3.9), there exists a constant $C>0$ depending only on $k_{-} / k_{+}, L_{2} / L_{1}$ but independent of $k_{ \pm}$and $\sigma$ such that

$$
\begin{aligned}
& \|\nabla \tilde{\varphi}\|_{L^{2}\left(\Omega_{\mathrm{PML}}\right)} \leq C \hat{C}^{-1} \alpha_{0}\left(1+k_{+} L_{1}\right)\|\varphi\|_{H^{1 / 2}\left(\Gamma_{B}\right)} \\
& \left\|A \nabla \overline{\tilde{\varphi}} \cdot \boldsymbol{n}_{1}\right\|_{H^{-1 / 2}\left(\Gamma_{B_{1}}\right)} \leq C \hat{C}^{-1} \alpha_{0}^{2}\left(1+k_{+} L_{1}\right)^{2}\|\varphi\|_{H^{1 / 2}\left(\Gamma_{B}\right)}
\end{aligned}
$$

where $\alpha_{0}=\max _{x \in \Gamma_{B_{1}}}\left(\left|\alpha_{1}\left(x_{1}\right)\right|,\left|\alpha_{2}\left(x_{2}\right)\right|\right)$, and $n_{1}$ is the unit outward normal vector on $\Gamma_{B_{1}}$.

Proof. For any $\zeta \in H^{1}\left(\Omega_{\mathrm{PML}}\right)$ such that $\zeta=\varphi$ on $\partial B$ and $\zeta=0$ on $\Gamma$, by (3.6) and the inf-sup condition in (3.7), we know that

$$
\hat{C}|||\tilde{\varphi}-\zeta| \|_{\Omega_{\mathrm{PML}}} \leq \sup _{0 \neq \psi \in H_{0}^{1}\left(\Omega_{\mathrm{PML}}\right)} \frac{|c(\tilde{\varphi}-\zeta, \psi)|}{|||\psi|||_{\Omega_{\mathrm{PML}}}}=\sup _{0 \neq \psi \in H_{0}^{1}\left(\Omega_{\mathrm{PML}}\right)} \frac{|c(\zeta, \psi)|}{\left.|||\psi|\right|_{\Omega_{\mathrm{PML}}}} .
$$

By Cauchy-Schwarz inequality, we get

$$
\left.|c(\zeta, \psi)| \leq C \alpha_{0}\left(1+k_{+} L_{1}\right)\|\zeta\|_{H^{1}\left(\Omega_{\mathrm{PML}}\right)}\right)|| \psi|\||_{\Omega_{\mathrm{PML}}} .
$$

Noting

$$
\left\|\left.|\zeta|\right|_{\Omega_{\mathrm{PML}}} \leq C \alpha_{0}\left(1+k_{+} L_{1}\right)\right\| \zeta \|_{H^{1}\left(\Omega_{\mathrm{PML}}\right)}
$$

by using the triangle inequality and the trace inequality, we conclude that

$$
\|\tilde{\varphi} \mid\| \Omega_{\Omega_{\mathrm{PML}}} \leq C \hat{C}^{-1} \alpha_{0}\left(1+k_{+} L_{1}\right)\|\varphi\|_{H^{1}\left(\Gamma_{B}\right)}
$$

which shows the first estimate in the theorem by using the definition of $\||| \cdot||_{\Omega_{\mathrm{PML}}}$.

Next, for any $\psi \in H^{1}\left(\Omega_{\mathrm{PML}}\right)$ such that $\psi=0$ on $\Gamma_{B}$, using (3.9a) and the integration by parts, we obtain

$$
\begin{aligned}
\int_{\Gamma}\left(A \nabla \overline{\tilde{\varphi}} \cdot \boldsymbol{n}_{\Gamma}\right) \bar{\psi} \mathrm{d} s & =\int_{\partial \Omega_{\mathrm{PML}}}\left(A \nabla \overline{\tilde{\varphi}} \cdot \boldsymbol{n}_{\Gamma}\right) \bar{\psi} \mathrm{d} s \\
& =\int_{\Omega_{\mathrm{PML}}}(A \nabla \overline{\tilde{\varphi}} \cdot \nabla \bar{\psi}+\nabla \cdot(A \nabla \overline{\tilde{\varphi}}) \bar{\psi}) \mathrm{d} \boldsymbol{x}=\int_{\Omega_{\mathrm{PML}}}\left(A \nabla \overline{\tilde{\varphi}} \cdot \nabla \bar{\psi}-k^{2} b \overline{\tilde{\varphi}} \bar{\psi}\right) \mathrm{d} x .
\end{aligned}
$$

It follows from the Cauchy-Schwarz inequality and (3.12) that

$$
\begin{aligned}
\left|\int_{\Gamma}\left(A \nabla \overline{\tilde{\varphi}} \cdot n_{\Gamma}\right) \bar{\psi} \mathrm{d} s\right| & \leq\left. C \alpha_{0}\left(1+k_{+} L_{1}\right)\||\tilde{\varphi}|\|\right|_{\Omega_{\mathrm{PML}}}\|\psi\|_{H^{1}\left(\Omega_{\mathrm{PML}}\right)} \\
& \leq C \hat{C}^{-1} \alpha_{0}^{2}\left(1+k_{+} L_{1}\right)^{2}\|\varphi\|_{H^{1}\left(\Gamma_{B}\right)}\|\psi\|_{H^{1}\left(\Omega_{\mathrm{PML}}\right)},
\end{aligned}
$$

which completes the proof after using the trace inequality. 


\subsection{Transparent boundary condition of the PML problem}

Now we introduce the approximate DtN operator $\mathscr{T}^{\mathrm{PML}}: H^{1 / 2}\left(\Gamma_{B}\right) \rightarrow H^{-1 / 2}\left(\Gamma_{B}\right)$ associated with the PML problem. Given $\xi \in H^{1 / 2}\left(\Gamma_{B}\right)$, let $\mathscr{T}^{\mathrm{PML}} \xi=\partial_{n} \varphi$ on $\Gamma_{B}$, where $\varphi \in H^{1}\left(\Omega_{\mathrm{PML}}\right)$ is the solution of (3.5).

Then the PML problem (3.3) is reduced to the following boundary value problem: Find $u^{\mathrm{PML}}$ such that

$$
\begin{aligned}
\Delta u^{\mathrm{PML}}+k^{2} u^{\mathrm{PML}}=0 & & \text { in } \Omega, \\
u^{\mathrm{PML}}=g & & \text { on } \Gamma_{D}, \\
{\left[u^{\mathrm{PML}}\right]=\left[\frac{\partial u^{\mathrm{PML}}}{\partial x_{2}}\right]=0 } & & \text { on } \Sigma \cap \Omega, \\
\partial_{n} u^{\mathrm{PML}}=\mathscr{T}^{\mathrm{PML}} u^{\mathrm{PML}} & & \text { on } \Gamma_{B} .
\end{aligned}
$$

The existence and uniqueness of the problem (3.13) is presented in [14]. Then the approximate DtN operator $\mathscr{T}^{\mathrm{PML}}$ is well-defined. The weak formulation of (3.13) is to find $u^{\mathrm{PML}} \in H^{1}(\Omega)$ such that $u^{\mathrm{PML}}=g$ on $\Gamma_{D}$, and

$$
a^{\mathrm{PML}}\left(u^{\mathrm{PML}}, v\right)=0, \quad \forall v \in H_{\Gamma_{D}}^{1}(\Omega),
$$

where the sesquilinear form $a^{\mathrm{PML}}: H^{1}(\Omega) \times H^{1}(\Omega) \rightarrow \mathbb{C}$ is defined by

$$
a^{\mathrm{PML}}(u, v)=\int_{\Omega}\left(\nabla u \cdot \nabla \bar{v}-k^{2} u \bar{v}\right) \mathrm{d} x-\int_{\Gamma_{B}}\left(\mathscr{T}^{\mathrm{PML}} u\right) \bar{v} \mathrm{~d} s .
$$

The following lemma establishes the relationship between the variational problem (3.14) and the weak formulation (3.4). The proof is straightforward based on our constructions of the transparent boundary conditions for the PML problem. The details of the proof is omitted for simplicity.

Lemma 3.3. Any solution $\hat{u}$ of the variational problem (3.4) restricted to $\Omega$ is a solution of the variational problem (3.14); conversely, any solution $u^{\mathrm{PML}}$ of the variational problem (3.14) can be uniquely extended to the whole domain to be a solution $\hat{u}$ of the variational problem (3.4) in $\Omega_{1}$.

For the completeness, here we present the estimate for the error $\mathscr{T} v-\mathscr{T}^{\mathrm{PML}_{v}}$ for any $v \in H^{1 / 2}\left(\Gamma_{B}\right)$, and the exponential convergence of PML solution.

Lemma 3.4. ([14, Lemma 7.1]) Let (H2) and $\gamma_{0} \bar{\sigma} \geq \max \left(k_{+}^{-1}, \min \left(d_{1}, d_{2}+L_{2}\right)\right)$ be satisfied. There exists a constant $C$ depending only on $\gamma_{0}, k_{-} / k_{+}, L_{2} / L_{1}$ but independent of $k_{ \pm}, L_{j}$, and $d_{j}, j=1,2$, such that, for any $v \in H^{1 / 2}\left(\Gamma_{B}\right)$,

$$
\left\|\mathscr{T}_{v-\mathscr{T}^{\mathrm{PML}}}\right\|_{H^{-1 / 2}\left(\Gamma_{B}\right)} \leq C\left(1+\hat{C}^{-1}\right) \gamma_{1}\left(1+k_{+} L_{1}\right)^{3} \alpha_{0}^{3}\left(1+\frac{\bar{\sigma}}{L_{1}}\right)^{2} e^{-k_{+} \gamma_{0} \bar{\sigma}}\|v\|_{H^{1 / 2}\left(\Gamma_{B}\right)},
$$

where $\gamma_{0}$ is define by (3.2), $\alpha_{0}$ is defined in Lemma 3.2, $\gamma_{1}:=e^{L_{2} \sqrt{k_{-}^{2}-k_{+}^{2}}}$. 
Theorem 3.1. ([14, Theorem 7.2]) Let (H1)-(H2) and $\gamma_{0} \bar{\sigma} \geq \max \left(k_{+}^{-1}, \min \left(d_{1}, d_{2}+L_{2}\right)\right)$ be satisfied. Let $u$ be the solution of (1.1a)-(1.1d). Then for sufficiently large $\bar{\sigma}$, the UPML problem (3.3) has a unique solution $\hat{u}$. Moreover, there exists a constant $C$ depending only on $\gamma_{0}, k_{-} / k_{+}$, $L_{2} / L_{1}$ but independent of $k_{ \pm}, L_{j}$, and $d_{j}, j=1,2$, such that

$$
\|u-\hat{u}\|_{H^{1}(\Omega)} \leq C\left(1+\hat{C}^{-1}\right) \gamma_{1}\left(1+k_{+} L_{1}\right)^{3} \alpha_{0}^{3}\left(1+\frac{\bar{\sigma}}{L_{1}}\right)^{2} e^{-k_{+} \gamma_{0} \bar{\sigma}}\|\hat{u}\|_{H^{1 / 2}\left(\Gamma_{B}\right)},
$$

where $\gamma_{0}$ is define by (3.2), $\alpha_{0}$ is defined in Lemma 3.2, and $\gamma_{1}$ is defined in Lemma 3.4.

\section{Finite element approximation}

In this section we introduce the finite element approximations of the PML problem (3.4), and develop the a posteriori error estimate, which is the basis of the adaptive finite element method.

Let $\mathcal{M}_{h}$ be a regular tetrahedral partition of the domain $\Omega_{1}$ such that $\left.\mathcal{M}_{h}\right|_{\Omega_{\mathrm{PML}}}$ and $\left.\mathcal{M}_{h}\right|_{\Omega}$ are also regular tetrahedral partitions of $\Omega_{\mathrm{PML}}$ and $\Omega$, respectively. Let $V_{h} \subset H^{1}\left(\Omega_{1}\right)$ be the conforming linear finite element space over $\Omega_{1}$, and

$$
V_{h, B_{1}}=\left\{v_{h} \in V_{h}: v_{h}=0 \text { on } \Gamma_{B_{1}}\right\}, \quad V_{h, 0}=\left\{v_{h} \in V_{h}: v_{h}=0 \text { on } \partial \Omega_{1}\right\} .
$$

The finite element approximation to the PML problem (3.4) reads as follows: Find $u_{h} \in$ $V_{h, B_{1}}$ such that $u_{h}=g$ on $\Gamma_{D}$, and

$$
b\left(u_{h}, v_{h}\right)=0, \quad \forall v_{h} \in V_{h, 0} .
$$

Theorem 4.1. For sufficiently small mesh size $h<h_{0}$, where $h_{0} \ll 1$ is a constant, the discrete problem (4.1) has a unique solution.

Proof. By Theorem 3.1, the continuous problem (3.3) has a unique solution $\hat{u}$. Then the sesquilinear form $b: H_{0}^{1}\left(\Omega_{1}\right) \times H_{0}^{1}\left(\Omega_{1}\right)$ satisfies the continuous inf-sup condition.

By the definition of $V_{h, 0}$, we know that $V_{h, 0}$ is dense in $H_{0}^{1}\left(\Omega_{1}\right)$ as the mesh size $h \rightarrow 0$. Using a general argument of Schatz [36], the following discrete inf-sup condition

$$
\sup _{0 \neq v_{h} \in V_{h, 0}} \frac{\left|b\left(u_{h}, v_{h}\right)\right|}{\left\|v_{h}\right\|_{V_{h, 0}}} \geq \hat{\gamma}\left\|u_{h}\right\|_{V_{h, 0}} \quad \forall v_{h} \in V_{h, 0}
$$

holds when the mesh size is sufficiently small, i.e., $h \ll 1$. Here the constant $\hat{\gamma}>0$ is independent of the mesh size. Then the general theory in [1, Chap. 5] implies that the discrete problem (4.1) has a unique solution. 


\subsection{Error representation formula}

Lemma 4.1 (Error representation formula). For any $\varphi \in H^{1}(\Omega)$, which is extended to be a function $\tilde{\varphi} \in H^{1}\left(\Omega_{1}\right)$ according to (3.9), and $\tilde{\varphi}_{h} \in V_{h, B_{1}}$, we have

$$
a\left(u-u_{h}, \varphi\right)=-b\left(u_{h}, \tilde{\varphi}-\tilde{\varphi}_{h}\right)+\int_{\Gamma_{B}}\left(\mathscr{T}-\mathscr{T}^{\mathrm{PML}}\right) u_{h} \bar{\varphi} \mathrm{d} s
$$

Proof. First by (2.4), (2.5), (3.14), and (3.15), we have

$$
a(u-\hat{u}, \varphi)=a^{\mathrm{PML}}(\hat{u}, \varphi)-a(\hat{u}, \varphi)=\int_{\Gamma_{B}}\left(\mathscr{T}-\mathscr{T}^{\mathrm{PML}}\right) \hat{u} \bar{\varphi} \mathrm{d} s .
$$

Then it yields that

$$
\begin{aligned}
a\left(u-u_{h}, \varphi\right)= & a(u-\hat{u}, \varphi)+a\left(\hat{u}-u_{h}, \varphi\right) \\
= & \int_{\Gamma_{B}}\left(\mathscr{T}-\mathscr{T}^{\mathrm{PML}}\right) \hat{u} \bar{\varphi} \mathrm{d} s+b\left(\hat{u}-u_{h}, \tilde{\varphi}\right)-\int_{\Gamma_{B}} \mathscr{T}\left(\hat{u}-u_{h}\right) \bar{\varphi} \mathrm{d} s \\
& -\int_{\Omega_{\mathrm{PML}}}\left(A \nabla\left(\hat{u}-u_{h}\right) \cdot \nabla \overline{\tilde{\varphi}}-k^{2} b\left(\hat{u}-u_{h}\right) \overline{\tilde{\varphi}}\right) \mathrm{d} x .
\end{aligned}
$$

Recalling that $n$ is the unit outer normal to $\Gamma_{B}$ which points outside $B$ and $n_{1}$ is the unit outer normal vector on $\Gamma_{B_{1}}$ directed outside $\Omega_{\mathrm{PML}}$, we deduce that

$$
\begin{aligned}
\int_{\Omega_{\mathrm{PML}}}\left(A \nabla\left(\hat{u}-u_{h}\right) \cdot \nabla \overline{\tilde{\varphi}}-k^{2} b\left(\hat{u}-u_{h}\right) \overline{\tilde{\varphi}}\right) \mathrm{d} x & =-\int_{\Gamma_{B}} \partial_{n} \overline{\tilde{\varphi}}\left(\hat{u}-u_{h}\right) \mathrm{d} s \\
& =-\int_{\Gamma_{B}}\left(\mathscr{T}^{\mathrm{PML}}\left(\hat{u}-u_{h}\right)\right) \bar{\varphi} \mathrm{d} s,
\end{aligned}
$$

where we have used (3.9a)-(3.9b), the definition of $\mathscr{T}^{\mathrm{PML}}$, and the identity (c.f., [13, Lemma 5.1])

$$
\int_{\Gamma_{B}}\left(\mathscr{T}^{\mathrm{PML}} \varphi\right) \bar{\psi} \mathrm{d} s=\int_{\Gamma_{B}}\left(\mathscr{T}^{\mathrm{PML}} \bar{\psi}\right) \varphi \mathrm{d} s, \quad \forall \varphi, \psi \in H^{1}\left(\Omega_{\mathrm{PML}}\right) .
$$

By (3.4), (4.1), and (4.4)-(4.5),

$$
\begin{aligned}
a\left(u-u_{h}, \varphi\right) & =b\left(\hat{u}-u_{h}, \tilde{\varphi}\right)+\int_{\Gamma_{B}}\left(\mathscr{T}-\mathscr{T}^{\mathrm{PML}}\right) u_{h} \bar{\varphi} \mathrm{d} s \\
& =-b\left(u_{h}, \tilde{\varphi}-\tilde{\varphi}_{h}\right)+\int_{\Gamma_{B}}\left(\mathscr{T}-\mathscr{T}^{\mathrm{PML}}\right) u_{h} \bar{\varphi} \mathrm{d} s,
\end{aligned}
$$

which completes the proof. 


\subsection{The a posteriori error analysis}

For any $K \in \mathcal{M}_{h}$, we denote by $h_{K}$ its diameter. Let $\mathcal{B}_{h}$ denote the set of all sides that do not lie on $\partial \Omega_{1}$. For any $e \in \mathcal{B}_{h}, h_{e}$ stands for its length. For any $K \in \mathcal{M}_{h}$, we introduce the residual:

$$
R_{K}:=\nabla \cdot\left(A \nabla u_{h}\right)+b k^{2} u_{h} \quad \text { for } K \in \mathcal{M}_{h} .
$$

For any interior side $e \in \mathcal{B}_{h}$ which is the common side of $K_{1}, K_{2} \in \mathcal{M}_{h}$, we define the jump residual across $e$ :

$$
J_{e}:=\left.\left(A \nabla u_{h}\right)\right|_{K_{1}} \cdot v-\left.\left(A \nabla u_{h}\right)\right|_{K_{2}} \cdot v \quad \text { for } e \in \mathcal{B}_{h},
$$

where we have used the notation that the unit normal vector $v$ on $e$ points from $K_{2}$ to $K_{1}$. For any $K \in \mathcal{M}_{h}$, we define the local error estimator $\eta_{K}$ as

$$
\eta_{K}:=\left(\left\|h_{K} R_{K}\right\|_{L^{2}(K)}^{2}+\frac{1}{2} \sum_{e \subset \partial K} h_{e}\left\|J_{e}\right\|_{L^{2}(e)}^{2}\right)^{1 / 2} .
$$

Theorem 4.2. There exists a constant $C>0$ depending only on $\gamma_{0}, k_{-} / k_{+}, L_{2} / L_{1}$ and the minimum angle of the mesh $\mathcal{M}_{h}$ such that the following a posterior error estimate holds

$$
\begin{aligned}
\left\|u-u_{h}\right\|_{H^{1}(\Omega)} \leq & C \hat{C}^{-1} \alpha_{0}\left(1+k_{+} L_{1}\right)\left(\sum_{K \in \mathcal{M}_{h}} \eta_{K}^{2}\right)^{1 / 2} \\
& +C \hat{C}^{-1} \gamma_{1}\left(1+k_{+} L_{1}\right)^{3} \alpha_{0}^{3}\left(1+\frac{\bar{\sigma}}{L_{1}}\right)^{2} e^{-k_{+} \gamma_{0} \bar{\sigma}}\left\|u_{h}\right\|_{H^{1 / 2}\left(\Gamma_{B}\right)} .
\end{aligned}
$$

Proof. Let $\Pi_{h}: H_{\Gamma_{B_{1}}}^{1}\left(\Omega_{1}\right) \rightarrow V_{h, B_{1}}$ be Scott-Zhang [37] interpolation operators satisfying the following interpolation estimates: For any $\varphi \in H^{1}\left(\Omega_{1}\right)$,

$$
\left\{\begin{array}{l}
\left\|\varphi-\Pi_{h} \varphi\right\|_{L^{2}(K)} \leq C h_{K}\|\nabla \varphi\|_{L^{2}(\tilde{K})^{2},} \\
\left\|\varphi-\Pi_{h} \varphi\right\|_{L^{2}(e)} \leq C h_{K}^{1 / 2}\|\nabla \varphi\|_{L^{2}(\tilde{e})^{2}}
\end{array} \quad \text { for } K \in \mathcal{M}_{h}\right.
$$

where $\tilde{K}$ and $\tilde{e}$ are the union of all elements in $\mathcal{M}_{h}$ having a non-empty intersection with $K \in \mathcal{M}_{h}$ and the side $e$, respectively.

Taking $\tilde{\varphi}_{h}=\Pi_{h} \tilde{\varphi} \in V_{h, B_{1}}$ in the error representation formula (4.2), we get

$$
a\left(u-u_{h}, \varphi\right)=-b\left(u_{h}, \tilde{\varphi}-\Pi_{h} \tilde{\varphi}\right)+\int_{\Gamma_{B}}\left(\mathscr{T}-\mathscr{T}^{\mathrm{PML}}\right) u_{h} \bar{\varphi} \mathrm{d} s=I_{1}+I_{2} .
$$

It follows from the integration by parts and (4.6)-(4.7) that

$$
I_{1}=\sum_{K \in \mathcal{M}_{h}}\left(\int_{K} R_{K}\left(\overline{\tilde{\varphi}-\Pi_{h} \tilde{\varphi}}\right) \mathrm{d} x+\frac{1}{2} \sum_{e \subset \partial K} \int_{e} J_{e}\left(\overline{\tilde{\varphi}-\Pi_{h} \tilde{\varphi}}\right) \mathrm{d} s\right)
$$


By (4.8) and the estimate (3.10), we have

$$
\begin{aligned}
\left|I_{1}\right| & \leq C\left(\sum_{K \in \mathcal{M}_{h}} \eta_{K}^{2}\right)^{1 / 2}\|\nabla \tilde{\varphi}\|_{L^{2}(\Omega)} \\
& \leq C \hat{C}^{-1} \alpha_{0}\left(1+k_{+} L_{1}\right)\left(\sum_{K \in \mathcal{M}_{h}} \eta_{K}^{2}\right)^{1 / 2}\|\varphi\|_{H^{1 / 2}\left(\Gamma_{B}\right)} .
\end{aligned}
$$

By Lemma 3.4, we have

$$
\left|I_{2}\right| \leq C \hat{C}^{-1} \gamma_{1}\left(1+k_{+} L_{1}\right)^{3} \alpha_{0}^{3}\left(1+\frac{\bar{\sigma}}{L_{1}}\right)^{2} e^{-k_{+} \gamma_{0} \bar{\sigma}}\left\|u_{h}\right\|_{H^{1 / 2}\left(\Gamma_{B}\right)}\|\varphi\|_{H^{1 / 2}\left(\Gamma_{B}\right)} .
$$

The proof is completed by using the above estimates in (4.9) and the inf-sup condition (2.6).

\section{Numerical experiments}

According to the discussion in Section 4, we choose the PML medium property as the power function and need to specify the thickness $d_{1}, d_{2}$ of the layers and the medium parameter $\bar{\sigma}$. It is clear to note from Theorem 4.2 that the a posteriori error estimate consists of two parts: the PML error $\epsilon_{\mathrm{PML}}$ and the finite element discretization error $\epsilon_{\mathrm{FEM}}$, where

$$
\begin{aligned}
& \epsilon_{\mathrm{FEM}}=\left(\sum_{K \in \mathcal{M}_{h}} \eta_{K}^{2}\right)^{1 / 2}, \\
& \epsilon_{\mathrm{PML}}=\gamma_{1}\left(1+k_{+} L_{1}\right)^{3} \alpha_{0}^{3}\left(1+\frac{\bar{\sigma}}{L_{1}}\right)^{2} e^{-k_{+} \gamma_{0} \bar{\sigma}}\left\|u_{h}\right\|_{H^{1 / 2}\left(\Gamma_{B}\right)} .
\end{aligned}
$$

In our implementation, we first choose $d_{1}, d_{2}$ and $\bar{\sigma}$ such that

$$
\gamma_{1}\left(1+k_{+} L_{1}\right)^{3} \alpha_{0}^{3}\left(1+\frac{\bar{\sigma}}{L_{1}}\right)^{2} e^{-k_{+} \gamma_{0} \bar{\sigma}} \leq 10^{-8}
$$

which makes the PML error (5.2) negligible compared with the finite element discretization error (5.1). Once the PML region and the medium property are fixed, we use the standard finite element adaptive strategy to modify the mesh according to the a posteriori error estimate. The adaptive FEM algorithm is summarized in Table 1.

In the following, we present three examples to demonstrate the competitive numerical performance of the proposed algorithm. The first-order linear element is used for solving the PML problem. Our implementation is based on Matlab PDE Toolbox.

In the three examples, the wave numbers of the two media are $k_{+}=1$ and $k_{-}=20$. We choose $\sigma=5$ for the medium property. Note that we can adjust the thickness of the PML layers such that the PML error is negligible compared with the finite element discretization error. 
Table 1: The adaptive FEM algorithm.

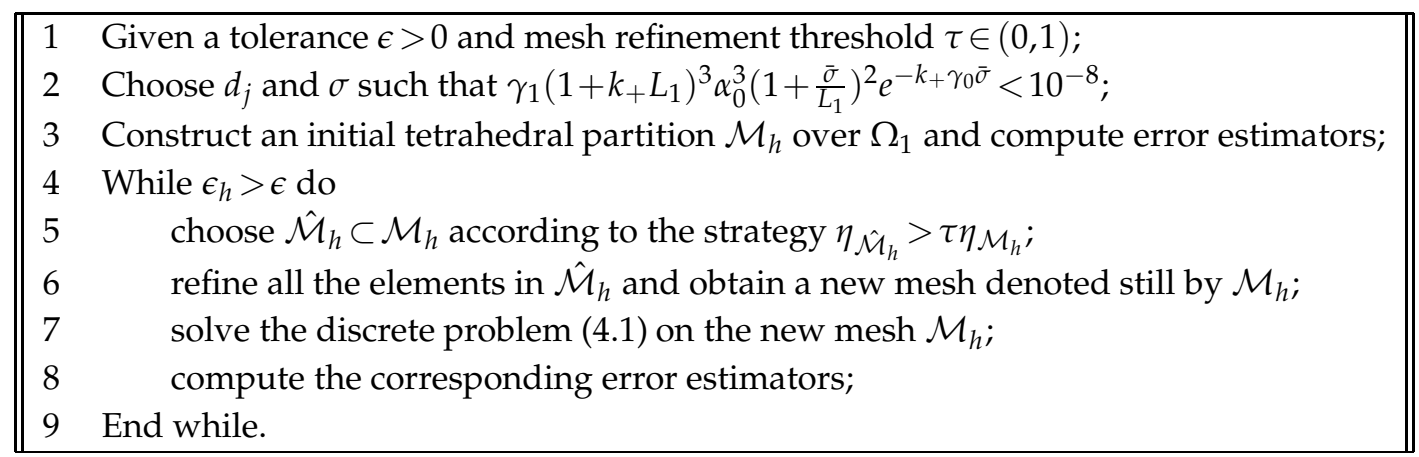

Example 5.1. Let the scatterer $D=(-0.2,0.2) \times(0.1,0.5)$, enclosed in the box $B=(-1,1) \times$ $(-1,1)$. We consider a scattering problem whose exact solution is known:

$$
u(x)= \begin{cases}\Phi\left(k_{+}, \boldsymbol{x}, \boldsymbol{y}\right)-\Phi\left(k_{+}, \boldsymbol{x}, \boldsymbol{y}^{\prime}\right)+\frac{\mathrm{i}}{2 \pi} \int_{-\infty}^{\infty} \frac{1}{\mu_{1}+\mu_{2}} e^{\mathrm{i} \xi\left(x_{1}-y_{1}\right)+\mathrm{i} \mu_{1}\left(x_{2}+y_{2}\right)} \mathrm{d} \xi, & x \in \mathbb{R}_{+}^{2}, \\ \frac{\mathrm{i}}{2 \pi} \int_{-\infty}^{\infty} \frac{1}{\mu_{1}+\mu_{2}} e^{\mathrm{i} \xi\left(x_{1}-y_{1}\right)+\mathrm{i}\left(\mu_{1} y_{2}-\mu_{2} x_{2}\right)} \mathrm{d} \xi, & x \in \mathbb{R}_{-}^{2},\end{cases}
$$

where $\mu_{1}=\left(k_{+}^{2}-\xi^{2}\right)^{1 / 2}, \mu_{2}=\left(k_{-}^{2}-\xi^{2}\right)^{1 / 2}, \boldsymbol{y}=(0,0.2)^{\top}$ is the source, and $\boldsymbol{y}^{\prime}=(0,-0.2)^{\top}$ is the image of $\boldsymbol{y}$. The thickness of the PML layers are chosen as $d_{1}=d_{2}=1$. Here $\Phi\left(k_{+}, \boldsymbol{x}, \boldsymbol{y}\right)$ is the Green function for the Helmholtz equation with constant wave number $k_{+}$, that is,

$$
\Phi\left(k_{+}, \boldsymbol{x}, \boldsymbol{y}\right)=\frac{\mathrm{i}}{4} H_{0}^{(1)}\left(k_{+}|\boldsymbol{x}-\boldsymbol{y}|\right)=\frac{\mathrm{i}}{4 \pi} \int_{-\infty}^{\infty} \frac{1}{\mu_{1}} e^{\mathrm{i} \xi\left(x_{1}-y_{1}\right)+\mathrm{i} \mu_{1}\left|x_{2}-y_{2}\right|} \mathrm{d} \xi .
$$

Fig. 3 displays the errors against the number of nodal points $N$ in $\Omega$. It clearly shows that the adaptive FEM yields quasi-optimal convergence rates, i.e.,

$$
\left\|u-u_{h}\right\|_{H^{1}(\Omega)}=\mathcal{O}\left(N^{-1 / 2}\right), \quad \eta_{h}=\mathcal{O}\left(N^{-1 / 2}\right),
$$

where $\eta_{h}$ is the a posterior error estimator. Fig. 4 plots the initial mesh with 1904 elements of the domain $\Omega_{1}$ (left) and the adaptive mesh with 20751 elements of $\Omega_{1}$ (right), respectively. From Fig. 4, we see that the mesh is much coarse away from the inner boundary since the solution decays exponentially in the PML, and the mesh in $\Omega \cap \mathbb{R}_{-}^{2}$ is very fine due to the large wave number $k_{-}=20$ compared with $k_{+}=1$ in $\mathbb{R}_{+}^{2}$. Fig. 5 shows the amplitude of the real part of $u_{h}$, which implies the solution decays very fast away from the inner boundary of the PML layer.

In the following two examples, the exact solutions are unknown. We compute the two examples by using both the uniaxial PML method and the circular PML method [11]. The complex coordinate stretching of the circular PML method is defined by

$$
\tilde{x}=x(1+\mathrm{i} \sigma(r-R)) \quad \text { for all } x \in \mathbb{R}^{2} \backslash \bar{B}_{R},
$$

where $r=|x|$ and $\sigma=5$. 


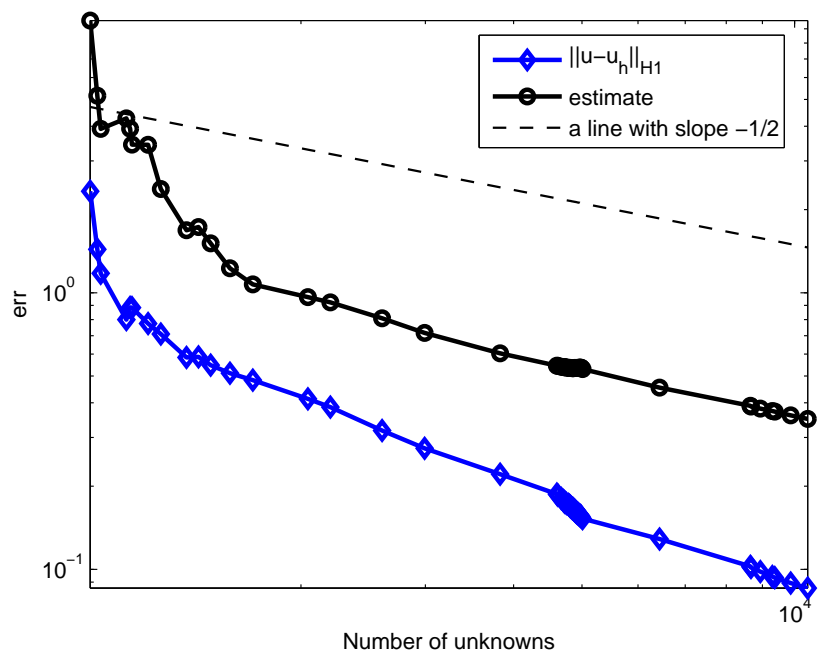

Figure 3: Example 5.1: Quasi-optimality of $H^{1}$ - error estimates and the a posteriori error estimates.
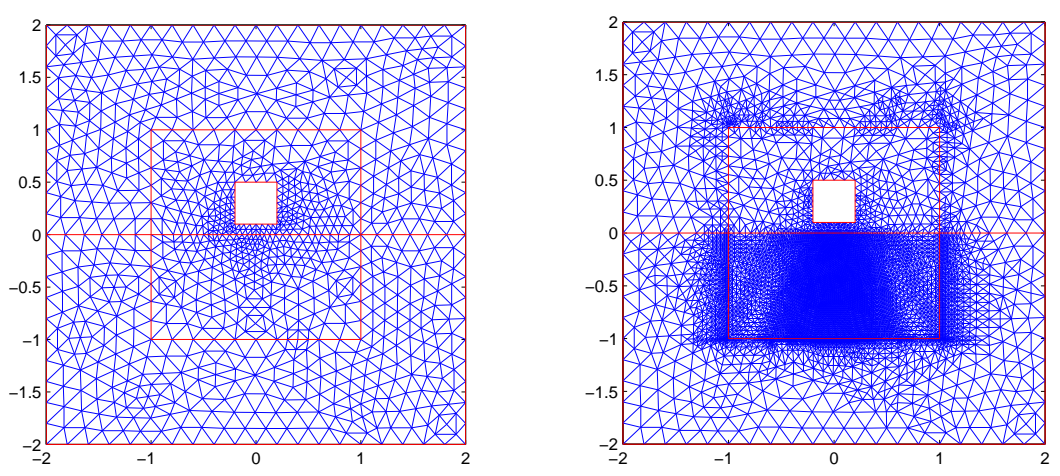

Figure 4: Example 5.1: The initial mesh and an adaptive mesh with 20751 elements of $\Omega_{1}$.

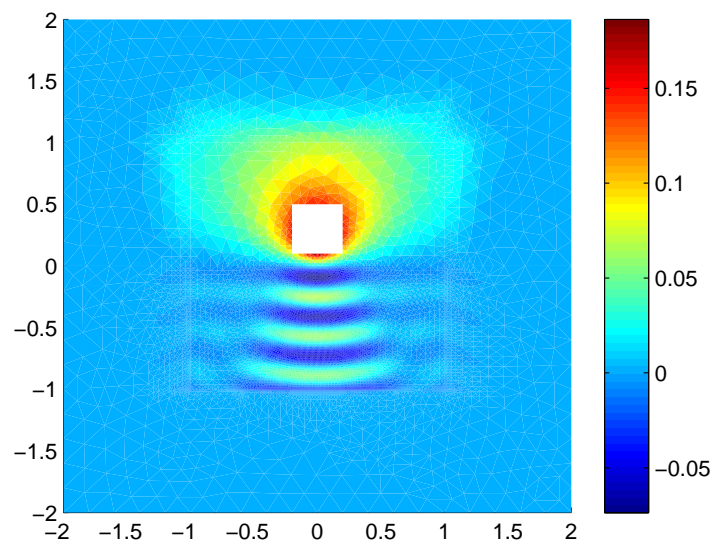

Figure 5: Example 5.1: The amplitude of the real part of $u_{h}$. 
Example 5.2. This example concerns the scattering of the incident plane wave $u^{\text {inc }}(x)=$ $e^{-\mathrm{i} k x_{1}}$ by a slim rectangular obstacle $D=(-1.5,1.5) \times(0.1,0.3)$ which is contained in the box $B=(-3,3) \times(-1,1)$. We choose $d_{1}=3$ and $d_{2}=1$ for the thickness of the uniaxial PML layers. For the circular PML method, we choose $R=L_{1}=3$ and the thickness of the PML layer $d=d_{1}=3$.

Fig. 6 shows that the quasi-optimality of the a posteriori error estimates holds for both the uniaxial PML method and the circular PML method. Table 2 compares the numerical results by using the two PML methods, which indicates that the uniaxial PML method is flexible for high aspect ratio scatterers.

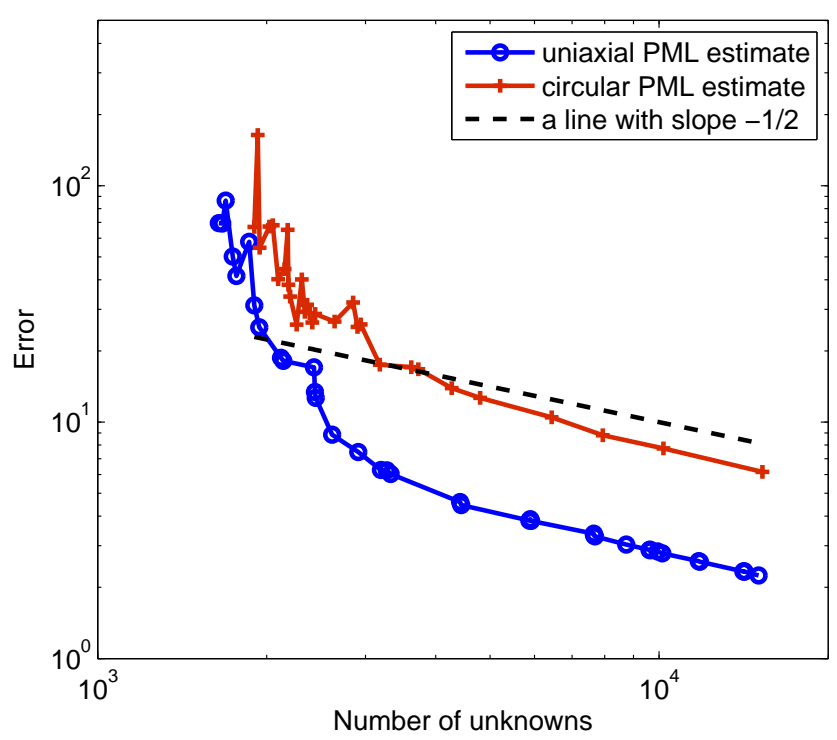

Figure 6: Example 5.2: Quasi-optimality of the a posteriori error estimates using both the uniaxial PML method and the circular PML method.

Table 2: Comparison of numerical results using the uniaxial PML method and the circular PML method for Example 2. DoF ${ }_{h}$ is the number of nodal points of mesh $\mathcal{M}_{h}$.

\begin{tabular}{||c|c||c|c||}
\hline \multicolumn{2}{||c||}{ Uniaxial PML method } & \multicolumn{2}{c||}{ Circular PML method } \\
\hline $\mathrm{DoF}_{h}$ & $\eta_{h}$ & $\mathrm{DoF}_{h}$ & $\eta_{h}$ \\
\hline 3326 & 6.0378 & 15277 & 6.1625 \\
\hline 4423 & 4.5894 & 25876 & 4.7140 \\
\hline 7648 & 3.3755 & 39392 & 3.7765 \\
\hline
\end{tabular}

Figs. 7 and 9 plot the adaptive meshes using the uniaxial PML method and the circular PML method, respectively. Figs. 8 and 10 show the amplitudes of the real part of $u_{h}$ using the uniaxial PML method and the circular PML method, respectively. We observe that the solutions using the two PML methods are consistent with each other. 


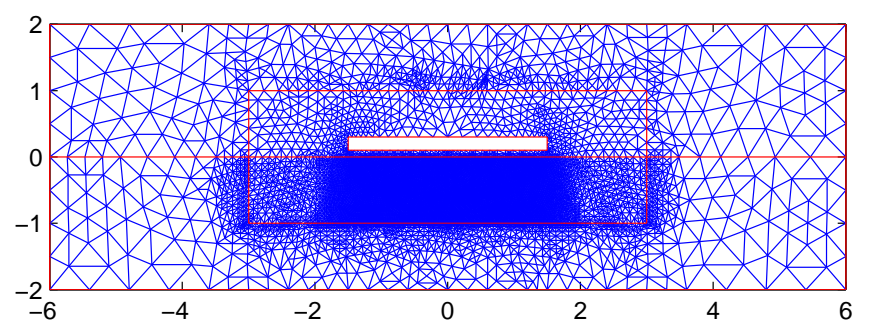

Figure 7: Example 5.2: An adaptive mesh with 29927 elements of $\Omega_{1}$ using the uniaxial PML method.

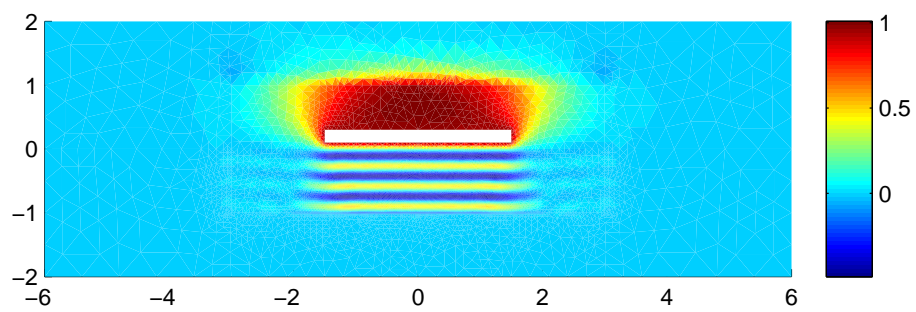

Figure 8: Example 5.2: The amplitude of the real part of $u_{h}$ using the uniaxial PML method.

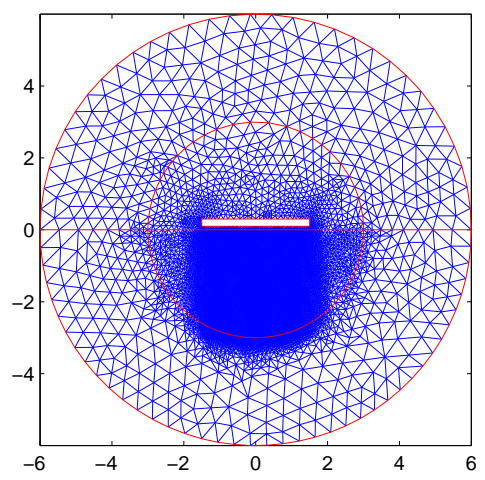

Figure 9: Example 5.2: An adaptive mesh with 30406 elements of $\Omega_{1}$ using the circular PML method.

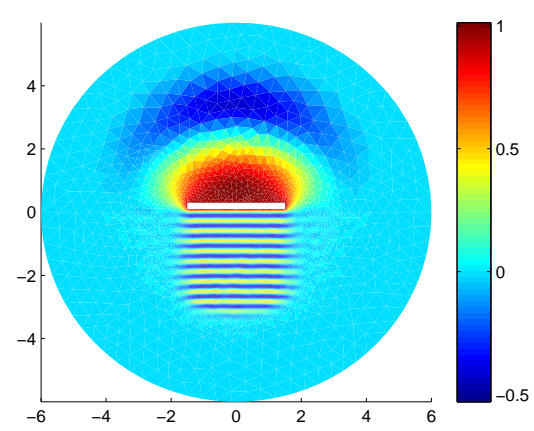

Figure 10: Example 5.2: The amplitude of the real part of $u_{h}$ using the circular PML method. 
Example 5.3. This example concerns the scattering of the incident plane wave $u^{\text {inc }}(x)=$ $e^{-\mathrm{i} k x_{1}}$ by two adjacent circular obstacles $D_{1}$ and $D_{2}$ centered at $c_{1}=(-0.2,-0.3)$ and $c_{2}=$ $(0.2,-0.3)$ with radius $r=0.2$. Clearly there is a strong singularity between the two circles in this example. The scatterers are contained in the box $B=(-1.5,1.5) \times(-1,1)$. We choose $d_{1}=1$ and $d_{2}=0.5$ for the thickness of the uniaxial PML layers. For the circular PML method, we choose $R=L_{1}=1.5$ and the thickness of the PML layer $d=d_{1}=1$.

Fig. 11 shows that the quasi-optimality of the a posteriori error estimates holds for both the uniaxial PML method and the circular PML method. It indicates that the two PML methods are comparable in solving this example.

Figs. 12 and 14 plot the adaptive mesh using the uniaxial PML method and the circular PML method, respectively. Figs. 13 and 15 show the amplitude of the real part of $u_{h}$

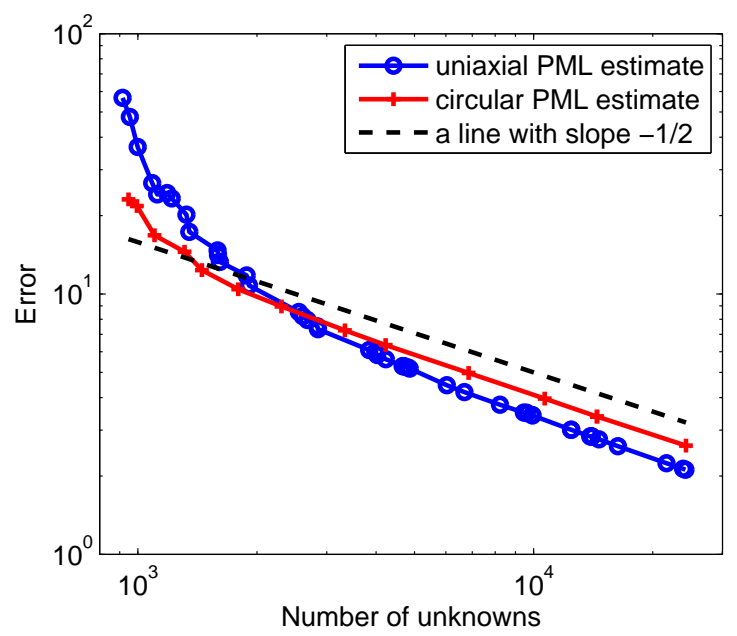

Figure 11: Example 5.3: Quasi-optimality of the a posteriori error estimates using both the uniaxial PML method and the circular PML method.

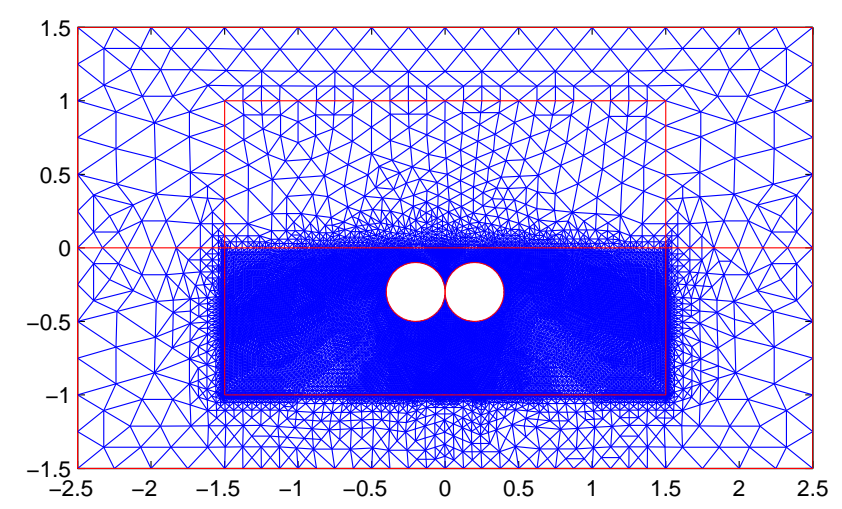

Figure 12: Example 5.3: An adaptive mesh with 48076 elements of $\Omega_{1}$ using the uniaxial PML method. 


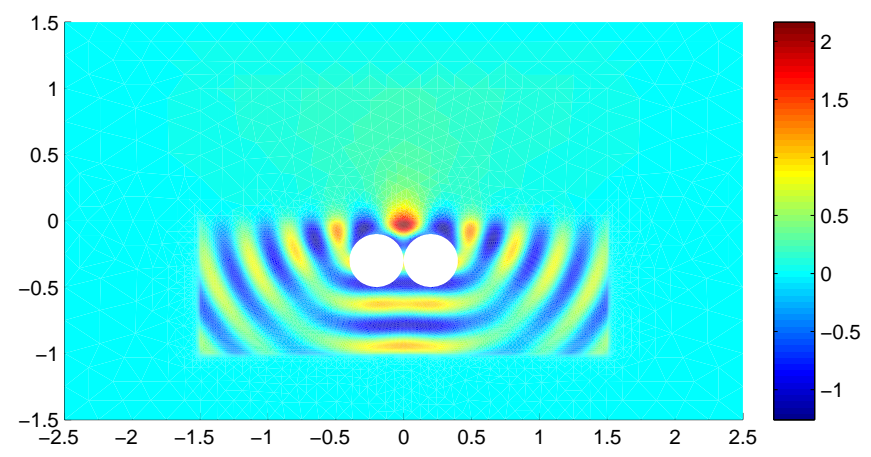

Figure 13: Example 5.3: The amplitude of the real part of $u_{h}$ using the uniaxial PML method.

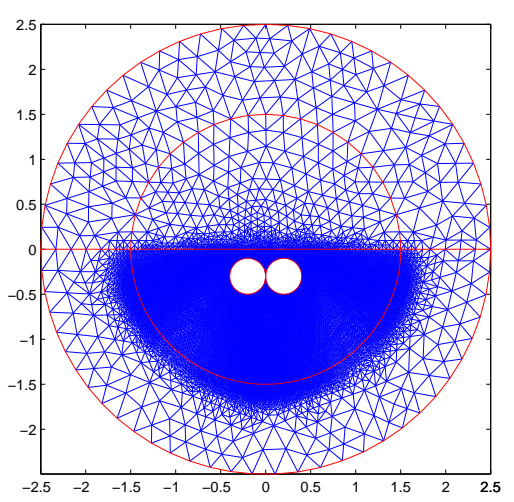

Figure 14: Example 5.3: An adaptive mesh with 48264 elements of $\Omega_{1}$ using the circular PML method.

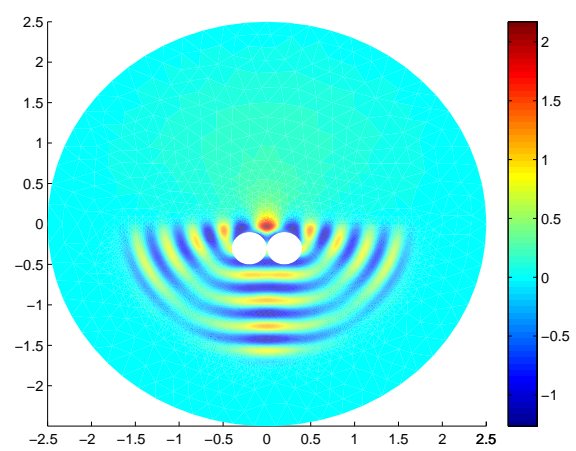

Figure 15: Example 5.3: The amplitude of the real part of $u_{h}$ using the circular PML method.

using the uniaxial PML method and the circular PML method, respectively. We observe that the solutions using the two PML methods are also consistent with each other for this example. 


\section{Acknowledgments}

The authors are grateful to Prof. Weiying Zheng for the insightful comments about our manuscript. The authors also thank the anonymous referees for their constructive suggestions and comments.

The research of XJ is supported by China NSF grants 11771057, 11401040 and 11671052. The research of JY is supported by China NSF grants 11671052, 11471052 and 91630202.

\section{References}

[1] I. Babuška and A. Aziz, Survey Lectures on Mathematical Foundations of the Finite Element Method, in The Mathematical Foundations of the Finite Element Method with Application to Partial Differential Equations, ed. by A. Aziz, Academic Press, New York, 1973, pp. 5-359.

[2] G. Bao and $\mathrm{H}$. Wu, Convergence analysis of the perfectly matched layer problems for timeharmonic Maxwell's equations, SIAM J. Numer. Anal., 43 (2005), pp. 2121-2143.

[3] J. P. Bérénger, A perfectly matched layer for the absorption of electromagnetic waves, J. Comput. Physics, 114 (1994), pp. 185-200.

[4] J.H. Bramble and J.E. Pasciak, Analysis of a finite PML approximation for the three dimensional time-harmonic Maxwell and acoustic scattering problems, Math. Comp., 76 (2007), pp. 597-614.

[5] J.H. Bramble and J.E. Pasciak, Analysis of a finite element PML approximation for the three dimensional time-harmonic Maxwell problem, Math. Comp., 77 (2008), pp. 1-10.

[6] J.H. Bramble, J.E. Pasciak and D. Trenev, Analysis of a finite PML approximation to the three dimensional elastic wave scattering problem, Math. Comp., 79 (2010), pp. 2079-2101.

[7] J.H. Bramble and J.E. Pasciak, Analysis of a Cartesian PML approximation to the three dimensional electromagnetic wave scattering problem, Int. J. Numerical Analysis and Modeling, 9 (2012), pp. 543-561.

[8] J.H. Bramble and J.E. Pasciak, Analysis of a Cartesian PML approximation to acoustic scattering problems in $\mathbb{R}^{2}$ and $\mathbb{R}^{3}$, J. Comput. Appl. Math., 247 (2013), pp. 209-230.

[9] S.N. Chandler-Wilde and P. Monk, The PML for rough surface scattering, Appl. Numer. Math., 59 (2009), pp. 2131-2154.

[10] J. Chen and Z. Chen, An adaptive perfectly matched layer technique for 3D time-harmonic electromagnetic scattering problems, Math. Comp., 77 (2008), pp. 673-698.

[11] Z. Chen and X. Liu, An adaptive perfectly matched layer technique for time-harmonic scattering problems, SIAM J. Numer. Anal., 43(2005), pp. 645-671.

[12] $\mathrm{Z}$. Chen and $\mathrm{H}$. Wu, An adaptive finite element method with perfectly matched absorbing layers for the wave scattering by periodic structures, SIAM J. Numer. Anal., 41(2003), pp. 799-826.

[13] Z. Chen and X. Wu, An adaptive uniaxial perfectly matched layer method for time-harmonic scattering problems, Numerical Mathematics: Theory, Methods and Applications, 1 (2008), pp. 113-137.

[14] Z. Chen and W. Zheng, Convergence of the uniaxial perfectly matched layer method for time-harmonic scattering problems in two-layer media, SIAM J. Numer. Anal., 48 (2010), pp. $2158-2185$.

[15] Z. Chen, X. Xiang, and X. Zhang, Convergence of the PML method for elastic wave scattering problems, Math. Comp., 85 (2016), pp. 2687-2714. 
[16] Z. Chen and W. Zheng, PML method for electromagnetic scattering problem in a two-layer medium, SIAM J. Numer. Anal., 55 (2017), pp. 2050-2084.

[17] W.-C. Chew, Waves and Fields in Inhomogenous Media, Van Nodtrand Reimhold, New York, 1990.

[18] W. Chew and W. Weedon, A 3D perfectly matched medium for modified Maxwell's equations with stretched coordinates, Microwave Opt. Techno. Lett., 13 (1994), pp. 599-604.

[19] D. Colton and R. Kress, Integral Equation Methods in Scattering Theory, John Wiley \& Sons, New York, 1983.

[20] J. Coyle and P. Monk, The Finite Element Approximation of Scattering in a Layered Medium, in Analytical and Computational Methods in Scattering and Applied Mathematics, F. Santosa and I. Stakgold, Eds., Chapman \& Hall/CRC, London. Research Notes in Mathematics number 417 (2000). pp. 67-84.

[21] M. Durán, R. Hein, and J. C. Nédélec, Computing numerically the Green's function of the half-plane Helmholtz operator with imedance boundary conditions, Numer. Math., 107 (2007), pp. 295-314.

[22] M. Durán, I. Muga, and J. C. Nédélec, The Helmholtz equation in a locally perturbed halfplane with passive boundary, IMA J. Appl. Math., 71 (2006), pp. 853-876.

[23] M. Durán, I. Muga, and J. C. Nédélec, The Helmholtz equation in a locally perturbed halfspace with passive boundary, Archive for Rational Mechanics and Analysis, 191 (2009), pp. 143-172.

[24] B. Engquist and A. Majda, Absorbing boundary conditions for the numerical simulation of waves, Math. Comp., 31 (1977), pp. 629-651.

[25] B. Engquist and A. Majda, Radiation boundary conditions for acoustic and elastic wave calculations, Comm. Pure and Appl. Math., 32 (1979), pp. 313-357.

[26] T. Hagstrom, Radiation boundary conditions for the numerical simulation of waves, Acta Numerica, 8 (1999), pp. 47-106.

[27] T. Hohage, F. Schmidt, and L. Zschiedrich, Solving time-harmonic scattering problems based on the pole condition. II: Convergence of the PML method, SIAM J. Math. Anal., 35 (2003), pp. 547-560.

[28] X. Jiang and W. Zheng, Adaptive perfectly matched layer method for multiple scattering problems, Comput. Methods Appl. Mech. Engrg., 201 (2012), pp. 42-52.

[29] X. Jiang, P. Li, J. Lv, and W. Zheng, An adaptive finite element PML method for the elastic wave scattering problem in periodic structure, ESAIM: Math. Model. Numer. Anal., 51 (2016), pp. 2017-2047.

[30] X. Jiang and P. Li, An adaptive finite element PML method for the acoustic-elastic interaction in three dimensions, Commun. Comput. Phys. 22 (2017), pp. 1486-1507.

[31] X. Jiang, P. Li, J. Lv, and W. Zheng, Convergence of the PML solution for elastic wave scattering by biperiodic structures, preprint.

[32] G. Kristensson, A uniqueness theorem for Helmholtz equation: penetrable media with an infinite interface, SIAM J. Math. Anal., 11(1980), pp. 1104-1116.

[33] M. Lassas and E. Somersalo, On the existence and convergence of the solution of PML equations, Computing, 60 (1998), pp. 229-241.

[34] M. Lassas and E. Somersalo, Analysis of the PML equations in general convex geometry, Proceeding of the Royal Society of Edinburg (2001), pp. 1183-1207.

[35] Chao Liang and Xueshuang Xiang, Convergence of an Anisotropic Perfectly Matched Layer Method for Helmholtz Scattering Problems, Numer. Math. Theor. Meth. Appl., 9 (2016), pp. 358-382. 
[36] A.H. Schatz., An observation concerning Ritz-Galerkin methods with indefinite bilinear forms, Math. Comp., 28 (1974), pp. 959-962.

[37] L. R. Scott and S. Zhang, Finite element interpolation of nonsmooth functions satisfying boundary conditions, Math. Comp., 54 (1990), pp. 483-493.

[38] F.L. Teixeira and W.C. Chew, Advances in the theory of perfectly matched layers, in: W.C. Chew et al, (eds.), Fast and Efficient Algorithms in Computational Electromagnetics, pp. 283-346, Artech House, Boston, 2001.

[39] E. Turkel and A. Yefet, Absorbing PML boundary layers for wave-like equations, Appl. Numer. Math., 27 (1998), pp. 533-557.

[40] D. Yu, The Natural Boundary Integral Method and Its Applications, Science Press \& Kluwer Academic Publishers, 2002.

[41] D. Zhang, F. Ma, and H. Dong, A finite element method with rectangular perfectly matched layers for the scattering from cavities, Journal of Computational Mathematics, 27 (2009), pp. 812-834. 